\title{
Upregulation of C-Reactive Protein by Placenta-Derived Mesenchymal Stem Cells Promotes Angiogenesis in A Rat Model with Cirrhotic Liver
}

\author{
Ji Hye Jun ${ }^{1}$, Jieun Jung ${ }^{2}$, Jae Yeon Kim ${ }^{1}$, Seong-Gyu Hwang ${ }^{3}$, Si Hyun Bae ${ }^{4}$, Gi Jin Kim ${ }^{1}$ \\ ${ }^{1}$ Department of Biomedical Science, CHA University, Seongnam, Korea \\ ${ }^{2}$ Non-Clinical Evaluation Center, CHA Advanced Research Institute, Seongnam, Korea \\ ${ }^{3}$ Department of Internal Medicine, CHA Bundang Medical Center, CHA University, Seongnam, Korea \\ ${ }^{4}$ Department of Internal Medicine, Catholic University Medical College, Seoul, Korea
}

\begin{abstract}
Background and Objectives: Liver cirrhosis is accompanied by abnormal vascular shunts. The Wnt pathway is essential for endothelial cell survival and proliferation. C-reactive protein (CRP), which is produced by hepatocyte, activates angiogenesis in cardiovascular diseases.

Methods and Results: The expression of CRP in $\mathrm{CCl}_{4}$-injured rat livers was detected using qRT-PCR and Western blotting after transplantation of placenta-derived mesenchymal stem cells (PD-MSCs) into rats. To determine whether CRP functions in hepatic regeneration by promoting angiogenesis through the Wnt pathway, we detected VEGF and $\beta$-catenin in liver tissues and $\mathrm{BrdU}$ and $\beta$-catenin in hepatocytes by immunofluorescence. The expression levels of $\mathrm{CRP}$, Wnt pathway-related and angiogenic factors were increased in $\mathrm{CCl}_{4}$-injured and PD-MSCs transplanted rat livers. In vitro, the expression levels of Wnt signaling and angiogenic factors were decreased in siRNA-CRP-transfected rat hepatocytes.

Conclusions: CRP upregulation by PD-MSCs participates in vascular remodeling to promote liver regeneration via the Wnt signaling pathway during hepatic failure.
\end{abstract}

Keywords: Liver cirrhosis, C-reactive protein, Placenta-derived mesenchymal stem cells, Wnt pathway, Vascular remodeling

\section{Introduction}

The liver is an essential organ for the metabolism for

Received: March 29, 2020, Revised: August 11, 2020,

Accepted: September 21, 2020, Published online: October 31, 2020 Correspondence to Gi Jin Kim

Department of Biomedical Science, CHA University, 335 Pangyo-ro, Bundang-gu, Seongnam 13488, Korea

Tel: +82-31-881-7145, Fax: +82-31-881-7249

E-mail: gjkim@cha.ac.kr

(c) This is an open-access article distributed under the terms of the Creative Commons Attribution Non-Commercial License (http://creativecommons.org/ licenses/by-nc/4.0/), which permits unrestricted non-commercial use, distribution, and reproduction in any medium, provided the original work is properly cited.

Copyright (c) 2020 by the Korean Society for Stem Cell Research lipids, proteins and carbohydrates, as well as the detoxification for endogenous metabolites (1). Liver sinusoidal endothelial cells (LSECs), which compose the hepatic vasculature, are in charge of important functions such as the proliferation of hepatocytes in the liver (2). LSECs releases angiocrine, including Wnt2 and hepatic growth factor (HGF), before the activation of angiogenesis and stimulate the regeneration of hepatocytes (3). Angiocrine signals induced by LSECs regulate the balance between fibrosis and hepatic regeneration (4). Hepatic failure involves diffused fibrosis, abnormal lobular architecture and the formation of intrahepatic vascular shunts (5). Therefore, vascular remodeling in liver tissues is a critical target for treating hepatic failure.

C-reactive protein (CRP) is known as the acute phase 
protein that is produced and released by hepatocytes in response to infection or tissue damage (6). CRP can be classified as monomeric CRP (mCRP) and pentameric CRP (pCRP) (7). Related to these isoforms of CRP, several studies have demonstrated pro-inflammatory and anti-inflammatory effects of $\mathrm{pCRP}$ and $\mathrm{mCRP}$, respectively (8). Recently, it has been shown that mCRP upregulates the tube-like formation signaling pathway in endothelial cells by activating protein C-ets-1 (ETS1), transcription factor and phosphorylating AKT, thereby increasing the release of chemokine ligand 2 (CCL2) (9). Additionally, together with oxidized low-density lipoprotein (oxLDL), CRP decelerates and stabilizes atherosclerotic progression by reducing the secretion of inflammatory factors such as TNF- $\alpha$ by macrophages (10). However, the regenerative effect of CRP has not yet been demonstrated in hepatic failure.

In the Wnt signaling, $\beta$-catenin is a key factor regulating transcriptional activity (11). Some studies have demonstrated that the inhibition of the Wnt pathway in the liver leads to pathophysiological events (12). The $\beta$ -catenin-dependent Wnt pathway is essential for EC survival and proliferation as well as the regulation of endothelial permeability (13). Accumulated evidence shows that endothelial $\beta$-catenin affects tight junctions, resulting in vascular integrity (14). However, the effect of $\beta$ -catenin related to vascular remodeling in liver cirrhosis is not yet clear.

Placenta-derived mesenchymal stem cells (PD-MSCs) have been characterized and have come to focus in stem cell research because of their proliferation, self-renewal, and low immunogenicity (15). In particular, transplanted PD-MSCs have been demonstrated to ameliorate $\mathrm{CCl}_{4}$-induced liver cirrhosis in mice (16). We previously reported that PD-MSCs have the ability to promote regeneration of hepatocyte via autophagic mechanism as well as induce IL-6/STAT3 signaling by reducing promoter methylation, further promoting the proliferation of hepatocytes in a $\mathrm{CCl}_{4}$-injured liver (17). Additionally, we demonstrated decreased CRP expression induces abnormal vascular structure in bile duct ligation rat model (18). However, the fundamental mechanism on vascular remodeling by CRP and their therapeutic potentials on hepatic diseases depend on PD-MSCs transplantation are still unclear.

We therefore aimed to investigate the expression of $\mathrm{CRP}, \beta$-catenin and angiogenic factors in a $\mathrm{CCl}_{4}$-injured rat liver model by PD-MSC transplantation and determine the regenerative effect of CRP through Wnt signaling and angiogenesis.

\section{Materials and Methods}

\section{Animals}

Six-week-old Sprague-Dawley rats were obtained (Orient Bio Inc.) and maintained in an air-conditioned facility. Hepatic failure was induced $\mathrm{CCl}_{4}$ (approximately $1.6 \mathrm{~g} / \mathrm{kg}$; Sigma-Aldrich) dissolved at $0.8 \mathrm{mg} / \mathrm{ml}$ in corn oil and intraperitoneally injected at $0.2 \mathrm{ml} / 100 \mathrm{~g}$ body weight twice a week for 3 weeks. Control rats $(n=5)$ were injected with an equal volume of corn oil alone. PD-MSCs $\left(2 \times 10^{6}\right.$ cells, $8 \sim 10$ passages) were injected into the tail vein of rats (TTX; $n=19)$. Non-transplanted (NTX; $n=19$ ) rats were maintained as sham controls. Liver tissues were harvested at 1, 2 and 3 weeks from rats in all groups. All animal experimental processes and the experimental protocol were approved by the Institutional Animal Care and Use Committee of CHA University, Seongnam, Korea (IACUC140009).

\section{Exosome precipitation from rat serum}

Equal amounts of serum from individual animals were pooled $(n=5)$. To isolate exosomes from rat serum, we used ExoQuick Exosome Precipitation solution (System Biosciences). The solution for exosome precipitation was used according to the manufacturer's instructions.

\section{Cell isolation and culture}

All participants provided written and informed consent prior to sample collection. Placental tissues were collected from women who had no medical, obstetrical, and surgical complications and who delivered at term (37 gestational weeks). The collection of samples and their use for research purposes were approved by the IRB of CHA General Hospital, Seoul, Korea (IRB 07-18). PD-MSCs were harvested as previously described. PD-MSCs were collected from the inner side of the chorionic membrane of the placenta. The cells scraped from the membrane were treated with $0.5 \%$ collagenase IV (Sigma-Aldrich) and cultured in Ham's F-12/Dulbecco's modified Eagle's medium (Invitrogen) supplemented with 10\% fetal bovine serum (FBS; Invitrogen), 1\% penicillin/streptomycin (Pen-Strep, Gibco), $25 \mathrm{ng} / \mathrm{ml}$ human fibroblast growth factor-4 (Peprotech), and $100 \mu \mathrm{g} / \mathrm{ml}$ heparin (Sigma-Aldrich). Rat epithelial cells (WB-F344s) were cultured in $\alpha$-MEM (Gibco) supplemented with 1\% Pen-Strep and 5\% FBS. Human umbilical vein endothelial cells (HUVECs) were cultured in endothelial cell growth medium (EGM)-2 SingleQuots (Lonza) at $37^{\circ} \mathrm{C}$ in a $5 \% \mathrm{CO}_{2}$ incubator. 


\section{Co-culture of WB-F344s, HUVECs, and PD-MSCs}

WB-F344s $\left(2 \times 10^{5}\right)$ and HUVECs $\left(6 \times 10^{4}\right)$ were seeded onto cover glasses (Marlenfeld GmbH \& Co.), and for double cell co-culture experiments, WB-F344s $\left(2 \times 10^{5}\right)$ were seeded onto cover glasses in a $100 \mathrm{~mm}$ culture plate (Corning). After 3 hours, the cells were exposed to $3 \mathrm{mM}$ $\mathrm{CCl}_{4}$ for 24 hours. The next day, the cells on cover glass were transferred to 6-well plates and co-cultured with PD-MSCs $\left(6 \times 10^{4}\right)$ were in Transwell inserts $(3 \mu \mathrm{m}$ pore).

\section{Magnetic cell sorting (MACS) analysis}

To sort co-cultured WB-F344s and HUVECs, we performed MACS analysis. Harvested WB-F344s and HUVECs were incubated with biotin-conjugated anti-CD31 (Miltenyi Biotec Inc.). Anti-biotin microbeads (Miltenyi Biotec Inc.) were reacted with the collected cells. The bound cells were sorted using MidiMACS Starting Kits (Miltenyi Biotec Inc.).

\section{Quantitative real-time PCR}

Total RNA was isolated from rat liver tissues and cells using TRIzol (Invitrogen). Reverse transcription was performed with 500 ng total RNA and Superscript III reverse transcriptase (Invitrogen). Real-time PCR was performed using SYBR EX Taq (Roche) and an Exicycler ${ }^{\mathrm{TM}} 96$ quantitative thermal block (Bioneer). PCR conditions were as follows: denaturation at $95^{\circ} \mathrm{C}$ for 15 minutes and $20 \mathrm{sec}-$ onds; 40 cycles at $95^{\circ} \mathrm{C}$ for 30 seconds; annealing at 52 $60^{\circ} \mathrm{C}$ for 40 seconds; extension at $70^{\circ} \mathrm{C}$ for 15 minutes; and a final extension at $72^{\circ} \mathrm{C}$ for 7 minutes. Gene expression was normalized to the expression of GAPDH. The sequences of the primers are as follows: rat CRP forward 5'- GCT TTT GGT CAT GAA GAC ATG TC -3', rat CRP reverse 5'- TCA CAT CAG CGT GGG CAT AG -3', rat Axin2 forward 5'- AAA CCT ATG CCT GTC TCC TC -3', rat Axin2 forward 5'- ATC CAC ACA TTT CTC CCT CT -3', rat VEGF forward 5'- ACG GAC AGA CAG ACA GAC AC -3', rat VEGF reverse 5'- CTT CTG GGC TCT TCT TCT CTC TC -3', rat albumin forward 5'- GCC CCA GAA CTC CTT TAC TA -3', rat albumin reverse 5'- AAT CTC TGC ATA CTG GAG CA -3', rat GAPDH forward 5'- TCC CTC AAG ATT GTC AGC AA -3', rat GAPDH reverse 5'- AGA TCC ACA ACG GAT ACA TT -3', human CRP forward 5'- TCG TAT GCC ACC AAG AGA CAA GAC A -3', human CRP reverse 5'- AAC ACT TCG CCT TGC ACT TCA TAC T -3', human Axin2 forward 5'- TCC CCA CCT TGA ATG AAG AA -3', human Axin2 reverse 5'- TGG TGG CTG GTG CAA AGA -3', human VEGF forward 5'- GCC TTG CCT TGC TGC TCT AC -3', human VEGF reverse 5'- ACA TCC ATG AAC
TTC ACC ACT TCG -3', human albumin forward 5'TGA GAA AAC GCC AGT AAG TGA C -3', human albumin reverse 5'- TGC GAA ATC ATC CAT AAC AGC -3', human GAPDH forward 5'- GCA CCG TCA AGG CTG AGA AC -3', and human GAPDH reverse 5'- GTG GTG AAG ACG CCA GTG GA -3'. All reactions were conducted in duplicate or triplicate. Relative mRNA expression was analyzed by the comparative CT method.

\section{Western blot}

Rat liver tissues and cells were lysed in RIPA buffer (Sigma-Aldrich) supplemented with protease inhibitor cocktail (Roche) and phosphatase inhibitor (Sigma-Aldrich). A total of $45 \mu \mathrm{g}$ protein was separated in sodium dodecyl sulfate polyacrylamide gels (SDS-PAGE). The separated proteins were transferred onto PVDF membranes (Bio$\mathrm{Rad})$. The membranes were incubated with anti-CRP (1 : 1,000 , Abcam Inc.), anti-VEGF (R\&D system), antiVEGFR2 (R\&D system), anti- $\beta$-catenin (active form, Cell Signaling), anti-pGSK3 (Cell Signaling), anti- $\beta$-catenin (active form, Cell Signaling), anti-albumin (Novus), antiHNF1 $\alpha$ (Abcam Inc.), anti-cyclinD1 (AbFrontier), antiactin (Sigma-Aldrich), anti-tubulin (Abcam Inc.), and antiGAPDH (AbFrontier) antibodies at $4^{\circ} \mathrm{C}$ over-night. The membranes were then incubated with horseradish peroxidase-conjugated secondary anti-mouse IgG (Cell Signaling), anti-rabbit IgG (Cell Signaling) or anti-goat IgG (Santa Cruz) antibody for 1 hour at RT. Bands were detected using Clarity Western ECL kit (Bio-Rad).

\section{Immunohistochemistry}

To observe the degree of inflammation in tissues following transplantation with PD-MSCs or not (NTX), we used anti-NF- $\kappa \mathrm{B}$ antibody. The sections were incubated in 3\% $\mathrm{H}_{2} \mathrm{O}_{2}$ in methanol to block endogenous peroxidase activity. After antigen retrieval, the slides were incubated with an anti-NF- $\kappa$ B antibody (Santa Cruz) at $4^{\circ} \mathrm{C}$ over-night, followed by an hour incubation with biotinylated secondary anti-rabbit antibody at room temperature (RT). Incubation with horseradish peroxidase-conjugated streptavidin-biotin complex (Dako) and 3,3-diaminobenzidine (EnVision Systems) was performed to generate a chromatic signal. The samples were counterstained with Mayer's hematoxylin. Additionally, the percentage of hepatocytes with NF- $\kappa$ B-positive nuclei relative to the total number of hepatocytes was counted in randomly selected sections (three fields per rat at $\times 400$ magnification). Images were detected using a Zeiss Axioskop 2 MAT microscope (Carl Zeiss MicroImaging). 


\section{Immunofluorescence}

To analyze the localization of VEGF and active $\beta$-catenin in liver tissues, we embedded rat livers in Tissue-Tek OCT compound (Sakura Finetechnical Co. Ltd.) and stored at $-80^{\circ} \mathrm{C}$. Cryosections of tissues were fixed in methanol (MERCK) and incubated in blocking solution (Dako). Anti-VEGF (Novus) and anti-active $\beta$-catenin (Cell Signaling) antibodies were used as primary antibodies at $4^{\circ} \mathrm{C}$ over-night. The secondary antibodies, Alexa 488 and 569 (Invitrogen), were incubated.

To analyze the localization of $\beta$-catenin and BrdU in rat hepatocytes, the WB-F344s co-cultured with PD-MSCs were fixed with $4 \%$ paraformaldehyde and permeabilized with $0.25 \%$ Triton $\mathrm{X}-100$ and $3 \% \mathrm{H}_{2} \mathrm{O}_{2}$. The cells were incubated with blocking solution (Dako) at RT and rabbit anti- $\beta$-catenin (Cell Signaling) and mouse anti-BrdU (Cell Signaling) antibodies at $4^{\circ} \mathrm{C}$ over-night. Subsequently, the cells were incubated with Alexa 568- or 488-conjugated secondary antibodies (Invitrogen) at RT. The slides were counterstained with DAPI. The images were observed with a fluorescence microscope EVOS (Thermo Fisher Scientific). All experiments were performed in triplicate.

\section{Enzyme-linked immunosorbent assay (ELISA)}

The concentrations of interleukin (IL)-6, IL-10 and CRP were determined by enzyme-linked immunosorbent assay (ELISA). Equal amounts of serum from individual animals or culture media were pooled $(n=5)$. All reactions were performed in triplicate. IL-6 and IL-10 concentrations were assayed using rat IL-6 and IL-10 ELISA kits
(R\&D system), according to the manufacturer's protocols. The concentration of CRP was measured using a rat C-Reactive Protein ELISA kit (Invitrogen) according to the manufacturer's instructions. The experiments were performed in triplicate.

\section{Statistical analysis}

Statistical significance was analyzed using Student's t-test with a significance level of $p<0.05$, and data were represented as the mean \pm standard deviation (SD). For multivariate data analysis, group differences were assessed with two-way ANOVA, followed by Tukey HSD test. All experiments were conducted in duplicate or triplicate.

\section{Results}

\section{The expression of inflammatory factors and CRP}

To evaluate the anti-inflammatory effects of PD-MSC transplantation, we analyzed NF- $\kappa \mathrm{B}$ expression in $\mathrm{CCl}_{4}$ injured rat livers using immunohistochemistry. As shown in Fig. $1 \mathrm{~A}$, in the $\mathrm{CCl}_{4}$-damaged NTX group, $\mathrm{NF}-\kappa \mathrm{B}$ was excessively localized in the nuclei for up to 3 weeks; however, transplantation of PD-MSCs resulted in a marked reduction in NF- $\kappa \mathrm{B}$ localization. Moreover, the number of NF- $\kappa$ B-positive cell was significantly lower in the TTX group than in the NTX group (*, p $<0.05$, Fig. 1B). Furthermore, we assayed the concentrations of IL-10, an anti-inflammatory factor, and IL-6, a regeneration factor, in the rat serum via ELISA. Compared to that in the NTX group, the expression of IL-10 in the rat serum was sig-

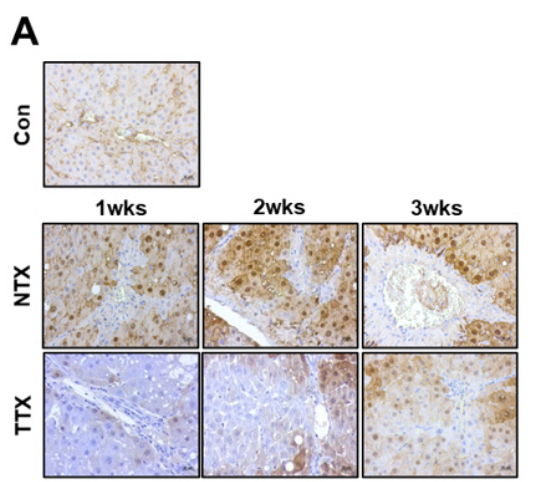

C

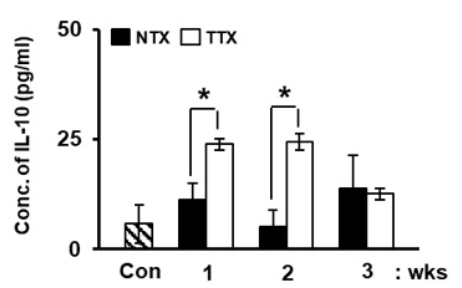

B

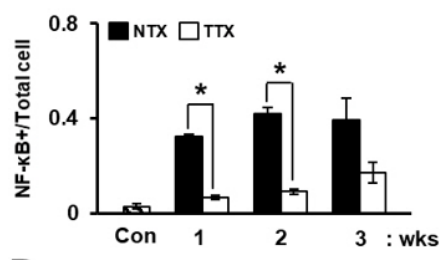

D

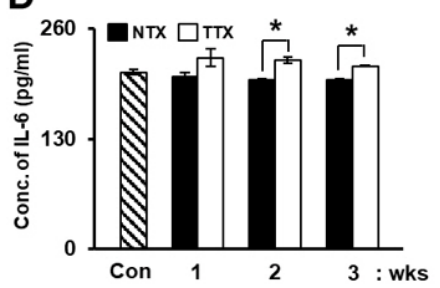

Fig. 1. PD-MSC transplantation attenuates inflammation in $\mathrm{CCl}_{4}$-injured rats. Expression of NF- $\kappa \mathrm{B}$ was detected by immunohistochemistry (A). $\times 400$, Scale bars; $20 \mu \mathrm{m}$. NF- $\kappa$ B-positive cells were quantified based on the total number of cells (B). Expression of IL-10 (C) and IL-6 (D) was analyzed in rat serum by ELISA. Data are represented as the triplicated mean \pm SD. $\mathrm{p}<0.05, *$; NTX vs.; NTX and TTX refer to the nontransplanted group and the PD-MSCtransplanted group, respectively. 
nificantly increased in the TTX group $\left(^{*}, \mathrm{p}<0.05\right.$, Fig. 1C). The secreted form of IL-6, which functions in hepatic regeneration, was also significantly increased in the TTX group (*, p $<0.05$, Fig. 1D).

Similar to IL-10 and IL-6, the expression of CRP in serum (Fig. 2A) and total liver protein (Fig. 2C) progressively increased in the TTX group compared to that in the control and NTX groups of in the $\mathrm{CCl}_{4}$-injured rat model. Additionally, CRP in exosomes isolated from serum was upregulated in the TTX group when normalized to heat shock protein (HSP)-70 and HSP-90, which are internal markers of exosomes (Fig. 2B and 2D). Therefore, PD-MSCs attenuate inflammatory responses and promote anti-inflammation through the induction of CRP expression.

\section{The correlation of Wnt signaling and angiogenesis factors in $\mathrm{CCl}_{4}$-injured rat liver}

To analyze the correlation between Wnt signaling and angiogenesis, we confirmed the expression and localization of the factors related to angiogenesis and Wnt signaling. Compared to those in the control and NTX groups, the factors related to angiogenesis, such as VEGF and VEGFR2, were significantly induced in the TTX group (*, $\mathrm{p}<0.05$, Fig. 3A, Supplementary Fig. S1A, and S1C). However, the expression of pGSK3, a Wnt inhibitory molecule, was significantly decreased in the TTX group compared to that in the control and NTX groups $(*, p<0.05$, Fig. 3B, Supplementary Fig. S1B). In contrast to GSK3, the active form of $\beta$-catenin was upregulated by PD-MSCs in $\mathrm{CCl}_{4}$-injured rat livers (Fig. 3B, Supplementary Fig. S1D). To investigate the localization and expression of VEGF and $\beta$-catenin, we performed immunofluorescence. VEGF and active $\beta$-catenin were localized in the cytoplasm and nuclei, respectively. Additionally, the expression of VEGF and $\beta$-catenin was higher in the control and TTX groups than in the NTX group (Fig. 3C). These results mean that activated $\beta$-catenin by PD-MSCs transplantation translocates into nucleus following promotes expression of VEGF in $\mathrm{CCl}_{4}$-injured rat livers. Further, to demonstrate the interaction between CRP, Wnt signaling, and angiogenesis in the liver, we analyzed the correlation between CRP, $\beta$-catenin, and VEGF of normalized fold change by band intensity of western blot. The correlation coefficient $\left(\mathrm{R}^{2}\right)$ of $\mathrm{CRP}$ and $\beta$-catenin was 0.8992 (Fig. 3D) as well as that of $\beta$-catenin and VEGF was 0.9295 (Fig. 3E). Therefore, these results represent that CRP, Wnt pathway, and vascular remodeling strongly correlate with one another in $\mathrm{CCl}_{4}$-injured rat liver.

\section{The correlation of CRP, Wnt signaling and angiogenic factors in rat hepatocytes}

To demonstrate the correlation among CRP, Wnt, and angiogenic and hepatic regeneration factors in hepatocytes, we transfected siRNA-CRP in rat hepatocytes and analyzed the mRNA and protein levels of CRP, albumin and factors related to Wnt signaling and angiogenesis. The expression of CRP, Axin2 (Fig. 4B), VEGF, and albumin (ALB) (Supplementary Fig. S2) mRNA was lower in siRNA-CRP-transfected hepatocytes than in non-trans-
A

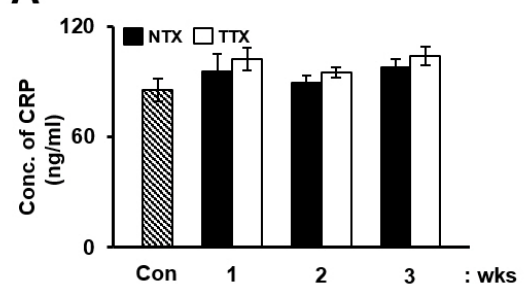

C
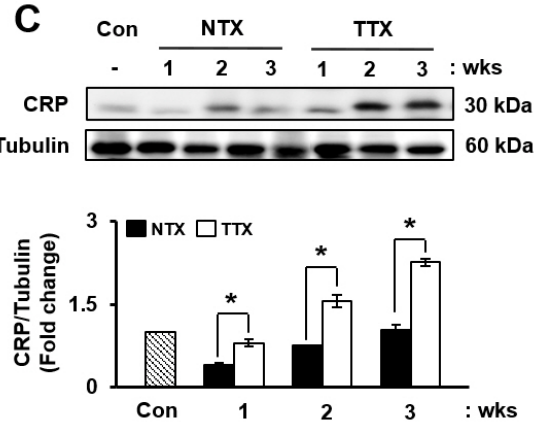

B



D
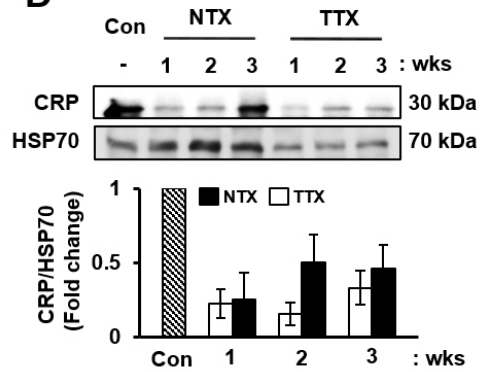

Fig. 2. PD-MSC transplantation improves the expression of CRP. Expression of CRP was analyzed by ELISA (A) and Western blotting from total proteins (C) and exosomes (B) and (D). Data are represented as the triplicated or duplicated mean \pm SD. SD; $\mathrm{p}<0.05$, *; NTX vs.; NTX and TTX refer to the non-transplanted group and the PD-MSC-transplanted group, respectively. 
A

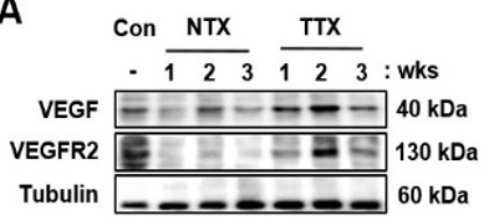

B



C
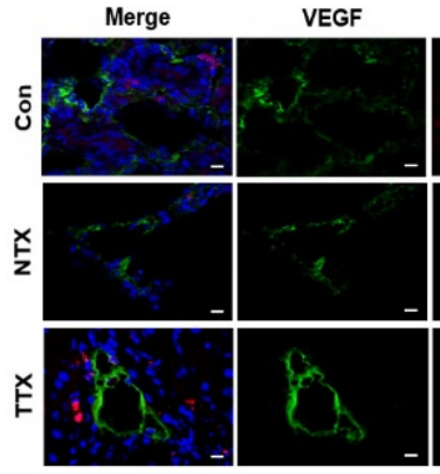

D



\section{B-catenin DAPI}



E

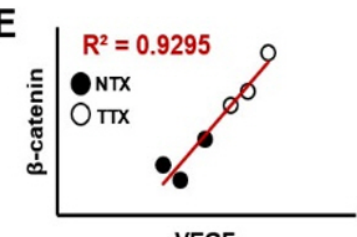

VEGF
Fig. 3. PD-MSC transplantation induces Wnt signaling and angiogenesis in $\mathrm{CCl}_{4}$-injured rats. Angiogenic (A) and Wnt signaling (B) factors were detected by Western blotting. Localization and expression of VEGF and $\beta$-catenin visualized using immunofluorescence $(C) . \times 630$, Scale bars; $20 \mu \mathrm{m}$. Positive correlations were found between CRP and $\beta$ catenin (D) also, between $\beta$-catenin and VEGF (E). NTX and TTX refer to the non-transplanted group and the PD-MSC-transplanted group, respectively.
A

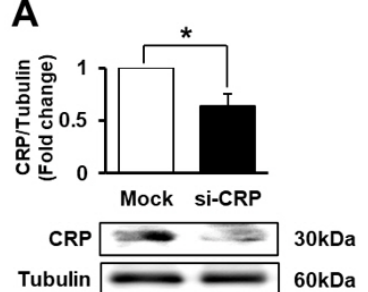

D



B

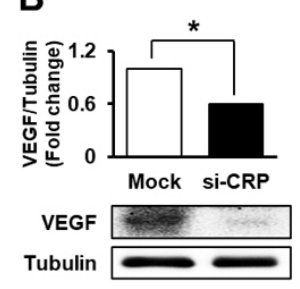

E

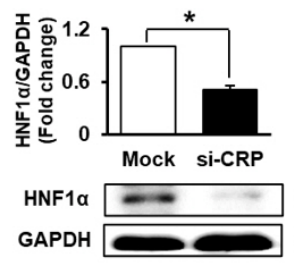

C

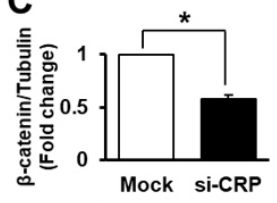

$40 \mathrm{kDa} \quad \beta$-catenin

$60 \mathrm{kDa}$ Tubulin

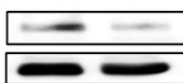

$97 \mathrm{kDa}$

$60 \mathrm{kDa}$

\section{$\mathbf{F}$}

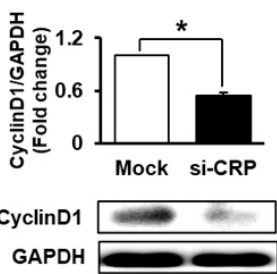

Fig. 4. CRP regulates angiogenesis and Wnt signaling in rat hepatocytes (WB-F344s). Protein levels of CRP (A), VEGF (B), $\beta$-catenin (C), ALB (D), HNF1 $\alpha$ (E), and CyclinD1 (F) were evaluated in siRNA-CRP-transfected rat hepatocytes by western blot. Data are represented as the triplicated mean \pm SD of. ${ }^{*}, \mathrm{p}<0.05$. Mock and si-CRP refer to non-transfected and siRNA-CRP-transfected rat hepatocytes, respectively. fected hepatocytes. As assessed by western blotting, CRP and VEGF protein expression was significantly suppressed via siRNA-CRP transfection (*, $\mathrm{p}<0.05$, Fig. $4 \mathrm{~A}$ and $4 \mathrm{~B})$. Additionally, the expression of $\beta$-catenin, a key factor in the Wnt pathway, was significantly inhibited by siRNACRP transfection in rat hepatocytes (*, p $<0.05$, Fig. 4C). Also, hepatic regeneration and proliferation factors such as ALB, hepatic nuclear factor 1 alpha $(\mathrm{HNF} 1 \alpha)$, and cyclinD1 were significantly repressed by siRNA-CRP transfection in WB-F344s (*, p<0.05, Fig. $4 \mathrm{D} \sim \mathrm{F})$. Therefore, CRP directly regulates angiogenesis through the Wnt pathway and promotes hepatic regeneration.

\section{BrdU incorporation and the expression of CRP and} $\beta$-catenin in rat hepatocytes co-cultured with PD-MSCs

To demonstrate the effect of CRP induced by PD-MSCs, we cultured $\mathrm{CCl}_{4}$-treated rat hepatocytes with PD-MSCs. The expression of CRP at both mRNA and 
protein levels was upregulated in rat hepatocytes by co-culture with PD-MSCs (Fig. 5A and 5B). To verify cell proliferation and localization of $\beta$-catenin, we performed immunofluorescence staining for $\operatorname{BrdU}$ and active $\beta$-catenin in rat hepatocytes that were co-cultured with PDMSCs post-CCl ${ }_{4}$ treatment. The localization of BrdU and $\beta$-catenin in rat hepatocytes is shown in Fig. 5C. The number of BrdU- or $\beta$-catenin-positive cells was significantly higher in the $\mathrm{CCl}_{4}$-treated and PD-MSC co-cultured group than in the non-co-cultured group $\left(^{*}, \mathrm{p}\right.$ $<0.05$, Fig. 5D). Also, the protein level of HNF1 $\alpha$ and cyclinD1, hepatic regeneration and proliferation markers, was dramatically decreased in $\mathrm{CCl}_{4}$ treated WB-F344s while their expression were recovered by PD-MSCs co-cultivation (*, $\mathrm{p}<0.05$, Fig. $5 \mathrm{E} \sim \mathrm{G}$ ). Therefore, CRP upregulation by PD-MSCs in rat hepatocytes activates Wnt signaling and facilitates hepatocyte regeneration.

\section{The correlation of CRP and angiogenic and Wnt signaling factors in hepatocytes and ECs co-cultured with PD-MSCs \\ To demonstrate the interaction between hepatocytes and} ECs via CRP and the Wnt signaling pathway, we used the culture scheme shown in Fig. 6A. The expression of CRP,
VEGF, Axin2 and albumin was analyzed in hepatocytes, WB-F344s, and ECs, HUVECs. In sorted hepatocytes and ECs, CRP mRNA was upregulated by co-culture with PD-MSCs (Fig. 6B and 6C). Additionally, the expression of VEGF and Axin2 was increased in $\mathrm{CCl}_{4}$-treated and PD-MSC co-cultured hepatocytes and ECs (Fig. 6D and 6E, Supplementary Fig. S3A and S3B). Furthermore, albumin, a hepatocyte marker, was upregulated in hepatocytes co-cultured with PD-MSCs (Supplementary Fig. S3C). However, albumin was not detected in the sorted ECs (Supplementary Fig. S3D). The expression of CRP protein, as detected by western blotting, was increased in the $\mathrm{CCl}_{4}$-treated and PD-MSC co-cultured rat hepatocytes (Supplementary Fig. S4A and S4B). Furthermore, the Wnt pathway (Supplementary Fig. S4A, S4C and S4E), angiogenesis (Supplementary Fig. S4A and S4F) and hepatic regeneration (Supplementary Fig. S4A and S4D) were activated in the co-cultured group. Comparing between only hepatocytes culture and EC cells and hepatocytes on co-culture systems, there was no difference between liver regeneration by expression of albumin. However, the difference between vascular regeneration and Wnt signaling factors was more dynamic on co-culture systems of EC cells and hepatocytes because the VEGF secretion is ex-

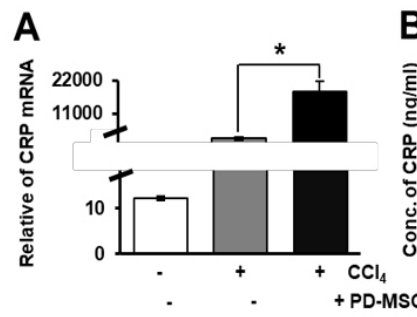

B
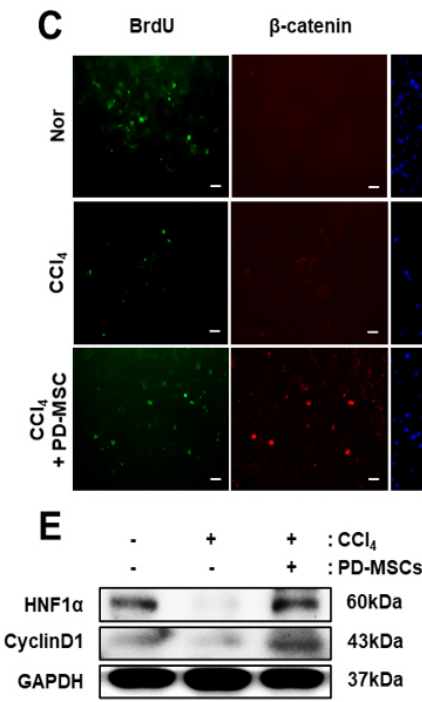

$\mathbf{F}$

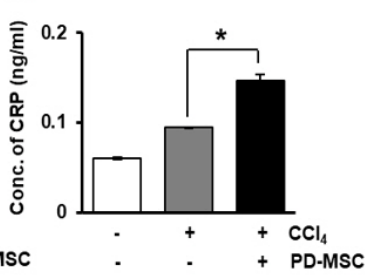

DAPI

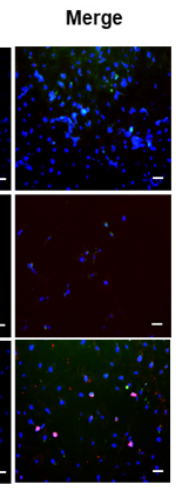

$F$

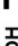

\section{离}


Fig. 5. PD-MSCs promote the expression of CRP and proliferation of hepatocytes through Wnt signaling. Expression of CRP was analyzed by qRT-PCR (A) and ELISA (B) after $\mathrm{CCl}_{4}$ treatment and co-culture with PDMSCs. Immunofluorescence staining shows the localization and expression of BrdU and $\beta$-catenin in rat hepatocytes treated with $\mathrm{CCl}_{4}$ and cocultured with PD-MSCs (C). $\times 400$, Scale bars; $20 \mu \mathrm{m}$. BrdU- or $\beta$-catenin- (D) positive rat hepatocytes were quantified after immunofluorescence staining. Protein levels of HNF1 $\alpha$, and CyclinD1 were detected by western blotting in rat hepatocytes treated with $\mathrm{CCl}_{4}$ and cocultured with PD-MSCs (E). Intensity of HNF1 $\alpha$ (F) and Cyclin D1 (G) protein bands was calculated. Triplicated data are represented as the mean \pm SD. ${ }^{*}, \mathrm{p}<0.05$. 
A



Fig. 6. PD-MSCs induce the expression of CRP and VEGF at the mRNA level. Schematic showing the experimental protocol (A). mRNA level of CRP (B), VEGF (D) was quantified in rat hepatocytes treated with $\mathrm{CCl}_{4}$ and co-cultured with HUVECs and PD-MSCs. mRNA level of CRP (C), VEGF (E) was assayed in HUVECs after $\mathrm{CCl}_{4}$ treatment and co-culture with rat hepatocytes and PD-MSCs. Duplicated data are represented as the mean \pm SD. ${ }^{*}, \mathrm{p}<0.05$.

ceptionally shown in the EC rather than the WB-F344. Therefore, we finally co-cultivated EC and hepatocytes to study hepatic regeneration by vascular regeneration and demonstrated CRP increased by PD-MSCs co-cultivation prompts vascular regeneration through Wnt signaling in hepatocytes by interacting with ECs.

\section{Discussion}

Hepatic cirrhosis is characterized by changes in nodule and hepatic sinusoidal re-capillarization (19). In addition to hepatic stellate cells, other non-parenchymal cells such as ECs are the major co-modulators of liver fibrosis (20). Angiogenic process by ECs is complicated and dynamic under physiological and pathological conditions (21). Recent studies have demonstrated that angiogenesis and sinusoidal remodeling are extensively correlated with hepatic fibrosis and regeneration (22). Therefore, we investigated the interaction of hepatocytes with ECs, through a direct co-culture system to determine the effects of this interaction on angiogenesis.

Mice with $\beta$-catenin knockout in the liver $(\beta$-catenin-LKO) revealed a significant role of $\beta$-catenin related to metabolic zonation, where $\beta$-catenin controls liver regeneration after hepatectomy by inducing cyclin-D1 (23). Similarly, Yang and colleagues made liver-specific Lrp $5 / 6$ knockout (Lrp-LKO) mice and showed that the Wnt pathway was limited in hepatocytes in the knockout model. Similar that in $\beta$-catenin-LKO mice, in Lrp-LKO mice, metabolic zonation was exacerbated by the lack of Cypla2 and Cyp2el. Additionally, Lrp-LKO resulted in a significant delay in liver regeneration owing to the lack of $\beta$ -catenin-TCF association and cyclin-D1 (24). Therefore, Wnt signaling in hepatocytes was identified as an important regulator of metabolic zonation and hepatic development. In another study, Leibing et al. (25) used endothelial subtype-specific Stab2-Cre driver mice to eliminate Wnt ligands from liver ECs to investigate the effects of the liver angiocrine Wnt pathway on liver growth and metabolic functions. The suppression of the angiocrine Wnt signaling pathway exacerbated metabolic zonation in the liver through the loss of $\beta$-catenin-dependent genes such as Axin2, glutamine synthase, and cytochrome P450 2E1. Similarly, through BrdU incorporation, we confirmed that increased endothelial markers and $\beta$-catenin can promote the proliferation of rat hepatocytes (Fig. 5).

Hepatic progenitor cells (HPCs) are a subtype of liver cancer stem cells that activate the Wnt/ $\beta$-catenin pathway and Notch signaling involved in angiogenesis. Notch and Hedgehog signaling pathways serve as powerful cancer-prevention mechanisms. Oishi et al. (26) demonstrated that $\beta$-catenin is upregulated in HPCs, which promotes vasculogenesis and maintains the stem cell niche. We found that upregulation of $\beta$-catenin and inhibition of pGSK3 by PD-MSCs activate vascularization through upregulating VEGF and VEGFR2 in vivo and in vitro (Fig. 3, 6, Supplementary Fig. S1, S4).

Our results indicated that the CRP is increased in the hepatic failure model in response to PD-MSC transplantation, correlating with IL-10 and anti-inflammatory factor expression. However, CRP has been established as an acute inflammatory marker because it has pattern-recognition activity. Although the serum level of CRP is associated with the risk and prognosis of several types of diseases, it is uncertain whether CRP is directly involved in disease or whether it is simply a bystander (27). In this study, we demonstrated a correlation between CRP and Wnt signaling in $\mathrm{CCl}_{4}$-treated rat livers transplanted PD-MSCs and rat hepatocytes co-cultured with PD-MSCs (Fig. 3D, Supplementary Fig. S4). Similar with our study, $\mathrm{Su}$ and colleagues identified mutations at SNP-286 (rs3091244) in the CRP promoter. Further analysis showed that the CRP mutations at SNP-286 co-occur with mu- 
tated adenomatous polyposis coli, which functions in the Wnt/ $\beta$-catenin pathway (28).

Pena and colleagues investigated the effects of CRP on angiogenic function in vascular ECs. mCRP activates the TF pathway in mouse ECs by phosphorylating AKT and promoting the expression of the transcription factor ETS1, leading to increased CCL2. Additionally, circulating pCRP, with mild pro-angiogenic effect via dissociation into mCRP on the surface of mouse ECs, can trigger potent angiogenic effects by enhancing the upregulation of the F3 gene and TF signaling (9). Chen et al. (29) confirmed that CRP affects the proliferation and pro-angiogenic paracrine activity of adipose-derived stem cells (ADSCs). In their study, CRP did not affect ADSC proliferation or apoptosis, but it induced ADSC migration by promoting the PI3K/AKT pathway. ADSCs treated CRP upregulated VEGF-A by inducing hypoxia inducible factor- $1 \alpha$, which enhanced tube formation on matrigel. Similarly, we found that CRP induction by PD-MSCs enhanced vascularization in $\mathrm{CCl}_{4}$-injured rat livers and hepatocytes co-cultured with ECs (Fig. 3, 6, Supplementary Fig. S4).

In conclusion, PD-MSCs increase the expression of CRP, which attenuates inflammation. The upregulated CRP by PD-MSCs activates Wnt signaling and angiogenesis and facilitates hepatocyte regeneration in vitro and in vivo. These results indicate that CRP induced by PD-MSCs is involved in vascular remodeling via the Wnt signaling during liver regeneration after hepatic failure and support the role of CRP as a hepatic regenerative factor.

\section{Acknowledgments}

This research was supported by a grant of the Korea Health Technology R\&D Project through the Korea Health Industry Development Institute (KHIDI), funded by the Ministry of Health \& Welfare, Republic of Korea (HI17C1050) and by Basic Science Research Program through the National Research Foundation of Korea (NRF) funded by the Ministry of Education, Science and Technology (2020M3A9B302618221).

\section{Potential Conflict of Interest}

The authors have no conflicting financial interest.

\section{Author Contributions}

J.H.J.: study concept and design, data acquisition, and drafting of the manuscript; J.J. and J.Y.K: data analysis; S.G.H. and S.H.B.: data interpretation, critical discussion; G.J.K.: conceived and designed the experiments and directed manuscript drafting, financial support and final ap- proval of manuscript.

\section{Supplementary Materials}

Supplementary data including four figures can be found with this article online at http://pdf.medrang.co.kr/paper/ pdf/IJSC/IJSC-13-s20052.pdf.

\section{References}

1. Rui L. Energy metabolism in the liver. Compr Physiol 2014;4:177-197

2. Poisson J, Lemoinne S, Boulanger C, Durand F, Moreau R, Valla D, Rautou PE. Liver sinusoidal endothelial cells: physiology and role in liver diseases. J Hepatol 2017;66:212227

3. Ding BS, Nolan DJ, Butler JM, James D, Babazadeh AO, Rosenwaks Z, Mittal V, Kobayashi H, Shido K, Lyden D, Sato TN, Rabbany SY, Rafii S. Inductive angiocrine signals from sinusoidal endothelium are required for liver regeneration. Nature 2010;468:310-315

4. Ding BS, Cao Z, Lis R, Nolan DJ, Guo P, Simons M, Penfold ME, Shido K, Rabbany SY, Rafii S. Divergent angiocrine signals from vascular niche balance liver regeneration and fibrosis. Nature 2014;505:97-102

5. Pinzani M, Rosselli M, Zuckermann M. Liver cirrhosis. Best Pract Res Clin Gastroenterol 2011;25:281-290

6. Salazar J, Martínez MS, Chávez-Castillo M, Núñez V, Añez R, Torres Y, Toledo A, Chacín M, Silva C, Pacheco E, Rojas J, Bermúdez V. C-reactive protein: an in-depth look into structure, function, and regulation. Int Sch Res Notices 2014;2014:653045

7. Eisenhardt SU, Habersberger J, Murphy A, Chen YC, Woollard KJ, Bassler N, Qian H, von Zur Muhlen C, Hagemeyer CE, Ahrens I, Chin-Dusting J, Bobik A, Peter K. Dissociation of pentameric to monomeric C-reactive protein on activated platelets localizes inflammation to atherosclerotic plaques. Circ Res 2009;105:128-137

8. Eisenhardt SU, Thiele JR, Bannasch H, Stark GB, Peter K. C-reactive protein: how conformational changes influence inflammatory properties. Cell Cycle 2009;8:3885-3892

9. Peña E, de la Torre R, Arderiu G, Slevin M, Badimon L. mCRP triggers angiogenesis by inducing $\mathrm{F} 3$ transcription and $\mathrm{TF}$ signalling in microvascular endothelial cells. Thromb Haemost 2017;117:357-370

10. Krayem I, Bazzi S, Karam M. The combination of CRP isoforms with oxLDL decreases TNF- $\alpha$ and IL- 6 release by U937-derived macrophages. Biomed Rep 2017;7:272-276

11. Clevers H. Wnt/beta-catenin signaling in development and disease. Cell 2006;127:469-480

12. Thompson MD, Monga SP. WNT/beta-catenin signaling in liver health and disease. Hepatology 2007;45:1298-1305

13. Korn C, Scholz B, Hu J, Srivastava K, Wojtarowicz J, Arnsperger T, Adams RH, Boutros $M$, Augustin HG, Augustin I. Endothelial cell-derived non-canonical Wnt li- 
gands control vascular pruning in angiogenesis. Development 2014;141:1757-1766

14. Tran KA, Zhang X, Predescu D, Huang X, Machado RF, Göthert JR, Malik AB, Valyi-Nagy T, Zhao YY. Endothelial $\beta$-catenin signaling is required for maintaining adult blood-brain barrier integrity and central nervous system homeostasis. Circulation 2016;133:177-186

15. Lee HJ, Jung J, Cho KJ, Lee CK, Hwang SG, Kim GJ. Comparison of in vitro hepatogenic differentiation potential between various placenta-derived stem cells and other adult stem cells as an alternative source of functional hepatocytes. Differentiation 2012;84:223-231

16. Zhang D, Jiang M, Miao D. Transplanted human amniotic membrane-derived mesenchymal stem cells ameliorate carbon tetrachloride-induced liver cirrhosis in mouse. PLoS One 2011;6:e16789

17. Jung J, Moon JW, Choi JH, Lee YW, Park SH, Kim GJ. Epigenetic alterations of IL-6/STAT3 signaling by placental stem cells promote hepatic regeneration in a rat model with CCl4-induced liver injury. Int J Stem Cells 2015;8:79-89

18. Jun JH, Choi JH, Bae SH, Oh SH, Kim GJ. Decreased C-reactive protein induces abnormal vascular structure in a rat model of liver dysfunction induced by bile duct ligation. Clin Mol Hepatol 2016;22:372-381

19. Xu B, Broome U, Uzunel M, Nava S, Ge X, KumagaiBraesch M, Hultenby K, Christensson B, Ericzon BG, Holgersson J, Sumitran-Holgersson S. Capillarization of hepatic sinusoid by liver endothelial cell-reactive autoantibodies in patients with cirrhosis and chronic hepatitis. Am J Pathol 2003;163:1275-1289

20. Iredale JP. Models of liver fibrosis: exploring the dynamic nature of inflammation and repair in a solid organ. J Clin Invest 2007;117:539-548

21. Djonov V, Baum O, Burri PH. Vascular remodeling by in- tussusceptive angiogenesis. Cell Tissue Res 2003;314:107117

22. Park S, Kim JW, Kim JH, Lim CW, Kim B. Differential roles of angiogenesis in the induction of fibrogenesis and the resolution of fibrosis in liver. Biol Pharm Bull 2015;38: 980-985

23. Yang J, Mowry LE, Nejak-Bowen KN, Okabe H, Diegel CR, Lang RA, Williams BO, Monga SP. $\beta$-catenin signaling in murine liver zonation and regeneration: a Wnt-Wnt situation! Hepatology 2014;60:964-976

24. Preziosi M, Okabe H, Poddar M, Singh S, Monga SP. Endothelial Wnts regulate $\beta$-catenin signaling in murine liver zonation and regeneration: a sequel to the Wnt-Wnt situation. Hepatol Commun 2018;2:845-860

25. Leibing T, Géraud C, Augustin I, Boutros M, Augustin HG, Okun JG, Langhans CD, Zierow J, Wohlfeil SA, Olsavszky V, Schledzewski K, Goerdt S, Koch PS. Angiocrine Wnt signaling controls liver growth and metabolic maturation in mice. Hepatology 2018;68:707-722

26. Oishi N, Yamashita T, Kaneko S. Molecular biology of liver cancer stem cells. Liver Cancer 2014;3:71-84

27. Moutachakkir M, Lamrani Hanchi A, Baraou A, Boukhira A, Chellak S. Immunoanalytical characteristics of C-reactive protein and high sensitivity C-reactive protein. Ann Biol Clin (Paris) 2017;75:225-229

28. Su HX, Zhou HH, Wang MY, Cheng J, Zhang SC, Hui F, Chen XZ, Liu SH, Liu QJ, Zhu ZJ, Hu QR, Wu Y, Ji SR. Mutations of C-reactive protein (CRP) -286 SNP, APC and p53 in colorectal cancer: implication for a CRP-Wnt crosstalk. PLoS One 2014;9:e102418

29. Chen J, Gu Z, Wu M, Yang Y, Zhang J, Ou J, Zuo Z, Wang J, Chen Y. C-reactive protein can upregulate VEGF expression to promote ADSC-induced angiogenesis by activating HIF-1 $\alpha$ via CD64/PI3k/Akt and MAPK/ERK signaling pathways. Stem Cell Res Ther 2016;7:114 